\title{
The Research of a Decision Making Framework for Marine Environmental Security
}

\author{
Pengfei Peng, Zekun Xie and Qiyuan Li \\ College of Electronic Naval Engineering University, Wuhan, China
}

\begin{abstract}
The disposal of marine emergencies and the maintenance of maritime rights are important links of marine environmental security guarantee. By analyzing its task process and characteristics, this paper analyzes its decision-making process, analyzes its operation needs, and then establishes a decision-making framework for collaborative guarantee and dynamic adjustment. According to the operation characteristics of decision-making process, a networked and intelligent decisionmaking technology framework and system function planning are designed, and the key technologies in the mission planning stage are analyzed. The framework of decision-making process and the technical framework of decision making have important reference value for marine environmental safety guarantee.
\end{abstract}

Keywords-maritime rights; marine emergencies; MDPSO; task decomposition

\section{INTRODUCTION}

Marine environmental safety refers to the safety of natural environment, resources development and maritime rights and interests in China's sea area, which is closely related to the comprehensive environmental safety of the country, and is related to the long-term stability and sustainable development of the country. In recent years, China's marine environmental security has been seriously challenged, and the threat to the marine environment is increasingly diversified: There was a fierce sovereignty dispute with the neighboring countries and the situation of the right to protect the rights was aggravated. With the expansion of maritime trade, pirate threats and terrorist threats are intensifying. Accidents such as collision and oil spill are frequent, which endangers the safety of life and property and pollute the ecological environment. In the face of the severe marine environment security situation, the abilities of disposing marine emergencies and the maintaining maritime rights need to be strengthened.

In view of the existing related technologies in China, most of the decision support systems are focused on the collection of static and dynamic information on ships and oceans in various jurisdictions. The situation is displayed on the electronic chart platform to provide information support for decision making, and the decision technology which is based on expert system and intelligent algorithm is not yet mature[1][2].Aiming at the disposal of marine emergencies and the maintenance of maritime rights, the system need to process the rich and complex marine information, which the need are high real-time, systematic and visualization .Merely by artificial judgment and decision making, it can not ensure the quick, timely, accurate and efficient decision. To build our future marine environmental security system, we must pay attention to the decision-making technology. Therefore, we analyze the main contents and characteristics of marine environmental security guarantee.

\section{The Decision-Making Process of Marine ENVIRONMENTAL SECURITY}

To optimize the decision-making process of marine environmental security, we must understand the contents and characteristics of their tasks. We first study the main contents of maritime emergency disposal and maritime rights protection, and analyze the decision-making process.

Maritime emergency disposal decision by accident alarm for specific events disposal decision triggered by the need to obtain the related information, such as marine environmental information, weather information, accident information, information disposal at sea power, and make analysis of event handling required personnel, supplies, power equipment and other needs, determine the disposal of events the task. According to analysis, the situation of resource assessment results by processing information, enter the task planning task planning stage, the main purpose is to determine the optimal matching of tasks and resources, the need to select the disposal area and constraint conditions, determine the disposal of resources [3].

Maritime rights decision making is a process based on the plan, which mainly includes the search and match of similar plans and the adjustment and the learning of the plan. Normalization of cruise maintenance has led the law enforcement departments to form a series of law enforcement plans, such as islands and reefs maritime sovereignty patrol, marine environmental protection, marine resources development and protection, monitoring foreign vessels and other plans. When making the plan of safeguarding rights, we need to match similar plans according to the needs of tasks, the information of the strength strength and the information of the marine environment, and get the alternatives. Combined with the situation at sea, we will evaluate and adjust the plan, and produce a feasible plan. At the same time, the scheme is studied and the plan library is updated.

\section{The TECHNICAL FrAmEWORK FOR MARINE ENVIRONMENTAL SECURITY SUPPORT}

The objects of maritime emergency disposal and maritime rights protection decisions are marine environment, support force and maritime targets. They are characterized by high complexity, large number and dispersed distribution. The constraint conditions include the constraints of the marine 
environment and physical conditions, the resource constraints of the support force, the constraints of the temporal and spatial conditions of tasks, and the movement and distribution constraints of the targets.

Therefore, decision making technology needs to solve the problem of ensuring the reasonable allocation of resources in the process of marine environmental security, and the accurate decision of disposing events. Combined with the characteristics of marine environmental security decision-making process, we need to build a decision-making technology framework for dealing with complex factors, rapid response and reasonable plan generation, and different steps need to cooperate with different technologies to achieve all kinds of business functions [4]. Integrating two decision processes, we can conclude that the decision-making process needs three stages, the information acquisition and situation assessment stage, the task planning stage, the program evaluation and the plan generation stage, as shown in Figure 1.

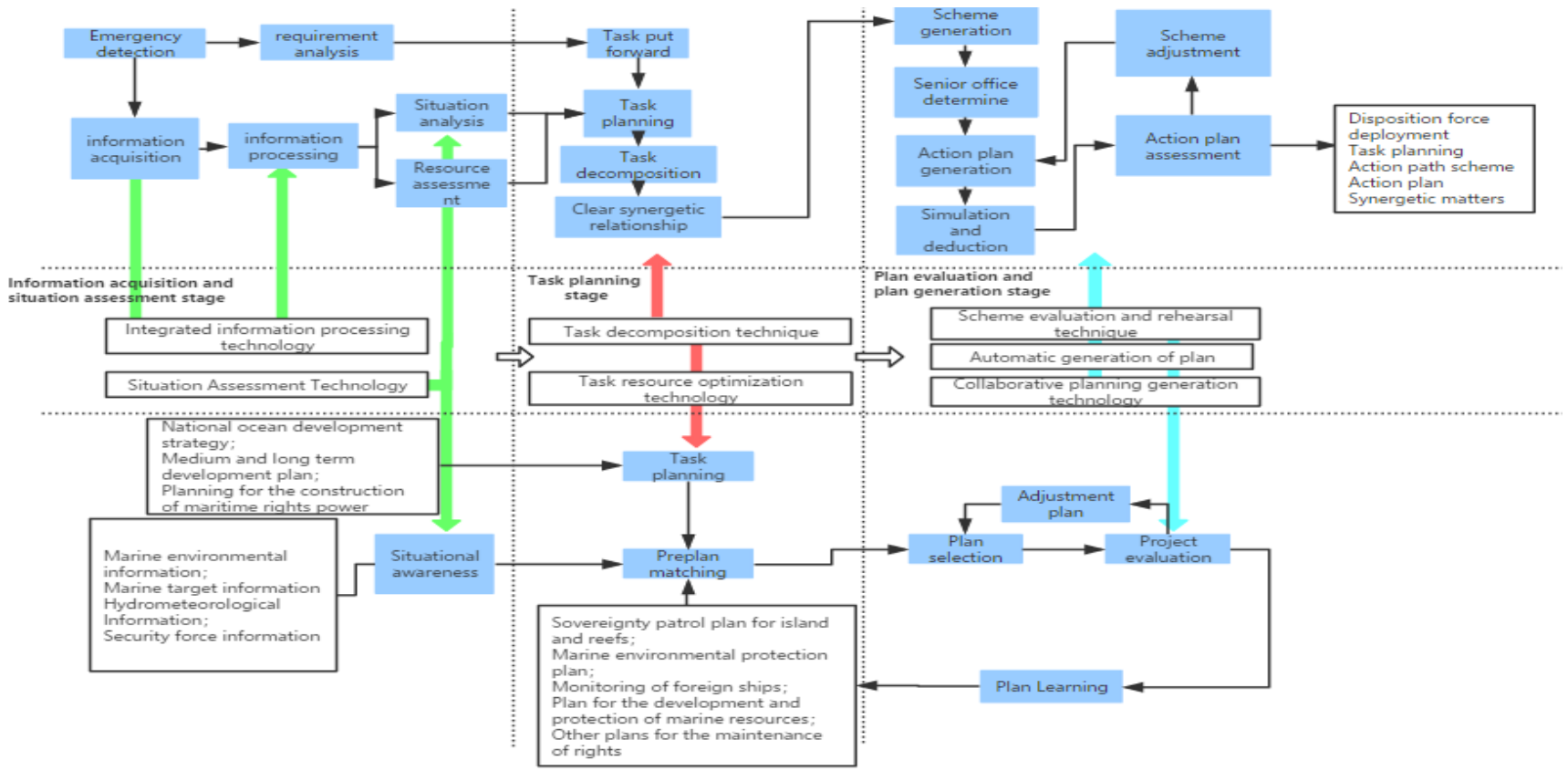

FIGURE I. THE TECHNICAL FRAMEWORK FOR MARINE ENVIRONMENTAL SECURITY SUPPORT

\section{AnAlysis of Key TeChNiCAL Methods In TASK PLANNING}

In view of the marine emergency handling and maritime rights protection tasks, the decision-making process is based on different characteristics, including intelligence acquisition and situation assessment, task planning, scheme evaluation and plan generation, and finally get the decision plan. The core technologies include information integrated processing technology, situation assessment technology, task decomposition technology, task resource optimization technology, program evaluation and deduction technology, plan automatic generation technology and collaborative planning generation technology. Before carrying out the marine environmental security task, in order to achieve the exact matching of tasks and resources, we need to plan the support tasks, decompose the tasks reasonably, evaluate the resources and capabilities of the support forces, and then match the sub tasks with the support forces. Therefore, task decomposition technology and task resource optimization are the key points in the research of decision-making process, shown as Figure 2.

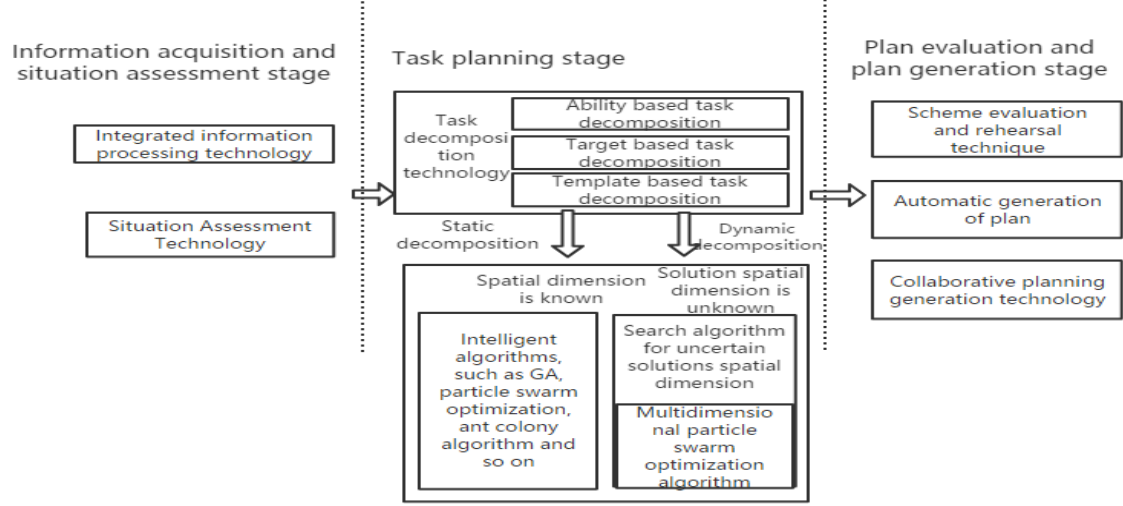

FIGURE II. DETAILED EXPLANATION OF THE TECHNICAL FRAMEWORK 


\section{A. Task Decomposition Technique}

In order to make the security forces to complete the task efficiently, we need to analyze the requirement of the task, get the task, and the need for situation analysis and to ensure the power capacity assessment, determine the resource space and time distribution, safeguard ability and other enforcement constraints for power resources tasks, and to determine the other related the constraint conditions, as the input of task planning[5]. Task decomposition is to achieve scientific and reasonable task planning, and also a part of program generation. Task decomposition has static decomposition and dynamic decomposition. Static decomposition refers to the decomposition that has been determined by task partition. Dynamic decomposition is the decomposition of task partition. Marine environmental security task complex according to different type of tasks and requirements, through the criteria and methods for the decomposition given security forces to cooperate to complete the task, or according to the execution unit ability of task decomposition, resource demand or given the completion of mission objectives, or in accordance with the task template matching of reasoning task decomposition, and then according to the estimated completion the task of time series, the meta mission, the formation of task chain [6].

\section{B. Task Decomposition Based on Capability}

In the protection of marine environment security, task resources include ship, maritime police forces navy navy and air force units, including all kinds of special task force to carry out the entity, satellite remote sensing and communications facilities, all kinds of sensors, and all kinds of professional team. One point of view of task decomposition and optimization of task resource matching is to start from resources and decompose the ability of task based resources. Ability is a quantitative description of the executive ability of task resources, whose description generally includes functional capabilities, speed and scope of task resources. The minimum task of executing resources according to their capacity is called meta task. Resource platform has one or more abilities. Capability is its inherent static attribute, and its ability set can be obtained through analyzing its structure function, and its meta task is determined[7].

\section{Task Decomposition Technology Based on Target}

Marine environmental security tasks have their specific security objectives, and the tasks to achieve their goals are aimed at certain goals (such as islands, sea areas, marine targets, etc.), and the mission is [9]. The task changes with the change of the state of the target, and the goal must correspond to the task. The task decomposition process, and a disposition to traverse the target based on the decomposition of sub tasks, the goal must be yuan or perform one task, based on meta task target decomposition is the smallest object by the analysis results, the target object attributes of resources and ensure the demand of the task of regional location, to be selected the task execution entity set and task priority information.

The task decomposition based on target matching needs to consider two aspects of the element task and the target sequence. The establishment of the target sequence requires the determination of the importance of the target on the basis of situation assessment and threat assessment. According to the principle of prioritize the most valuable goals, we establish relevant task sequences. At the same time, considering the cooperative relationship of multiple objectives, we can solve the problem from the perspective of target package. The target package is a set of target particles combined according to certain rules, which is often related to typical collaborative execution style.

\section{Task Decomposition Based on Task Template}

On the basis of complex tasks and meta tasks, complex templates divide operational tasks into 3 levels of [9]: tactical action layer, basic task layer and joint task layer. Tactical action, corresponding military activities in basic tactical operations, the lowest layer is located in the task hierarchy, used to construct the other levels of the task. This layer by layer decomposition of the tree structure is determined by the target situation and task pattern involved in the task. When a task needs to be decomposed, it is first matched by the hierarchical network and the existing template. The corresponding template is selected from the template library according to its similarity, and its matching result is the decomposed meta task and the set of task sequence. The decomposition method of step by step task is to generate a series of related new tasks by refining the operational objectives and analyzing mission requirements, so that the task decomposition is diversified.

\section{E. Tasks-Resources Optimization Technology}

The ultimate goal of task planning is to achieve the optimal matching of the meta task and the guarantee resources. The process involves multiple objective programming problems. In view of the uncertain dimension of the solution space, the traditional heuristic algorithm is slightly deficient. Based on that, many algorithms for solving the spatial dimension of the uncertain solution are proposed. [10] is a typical multi particle swarm optimization method to solve the optimal solution of optimization problem where the optimal dimensions of the unknown, the basic PSO algorithm and the main disadvantages of many other variants of the PSO algorithm is used only for the solution space search dimension is fixed, and the dynamic optimization problem of the optimal solution of the optimal degree is not necessarily the task resources need to be determined in the process of PSO. Compared with the conventional particle swarm algorithm, particle swarm multidimensions PSO, with global fractal improved global optimal solution of peak shift optimal construction technology, with the help of the optimization function to evaluate the optimal dimensional acquisition, the global convergence is improved, thus to achieve the optimal solution under the condition of uncertain dimensions.

\section{CONCLUSION}

Through the business process analysis and disposal of maritime rights emergencies at sea, and discuss the plan type and reaction type decision process, summed up the related technical framework of the discussion to improve the decision efficiency and decision accuracy, and introduces the technology and task resource optimization technology in the decision-making process of task decomposition provides an important reference for the construction of marine environmental security system. 


\section{REFERENCES}

[1] Yonghong Luo, Study of Intelligentized Aided Decision System for Salvage and Rescue at Sea, NAVIGATION OF CHINA,2004(3):21-24.

[2] LIANG Xiao-guang; CHEN Jun, Overall Architecture of Ocean Rightsafeguard Information System, Radio Communications Technology ,2014(02)

[3] CHENG Kai;CHEN Gang;ZHANG Pin, Review on Methods of Operation Planning, Computer Science ,2016(12)

[4] ZENG Bin; YAO Lu; HU Wei; YANG Guang, Scheduling algorithm for maintenance tasks under uncertainty, Systems Engineering and Electronics ,2016(03)

[5] XIAO Yi-hong 1,ZHOU Xian-zhong 1,ZHANG Tie, Mission Decomposition Tactics with Variable Granularity Based on Extended Hierarchical Task Network Planning, Fire Control \& Command Control ,2011(07)

[6] LIU Hong-fang 1,YANG Dong-sheng, Research on Methodology of Generating Task-Based Tactical Situation Picture, Computer Simulation ,2006(07)

[7] YANG Shi-xing1,YANG Dong-sheng1,ZHANG Wei-ming2,Research on Method of Mission Decomposition and Task Modeling, Fire Control and Command Control , 2009(08)

[8] LIU Hong-fang1, YANG Dong-sheng2, LIU zhong1, ZHANG Weiming, Research on Methodology of Resource Tailoring and Clustering in Battlefields, Journal of System Simulation ,2006(09)

[9] WANG Tao; SHI Zhan; ZHAO Yu-lin, Swift Generation Technology of Operational Plan with Variable Granularity Based on Hierarchical Task Network, Command Control \& Simulation ,2015(01)

[10] Kiranyaz S, Ince T, Gabbouj M. Multidimensional Particle Swarm Optimization for Machine Learning and Pattern Recognition. Springer Berlin Heidelberg, 2014. 